\title{
THE GRAPH OF DIAGRAM GROUPS FROM DIRECT PRODUCT OF THREE FREE SEMIGROUPS
}

\author{
Sri Gemawati and Abd. Ghafur Bin Ahmad
}

\begin{abstract}
In this paper, we will discuss the diagram groups from direct product of three free semigroups $[X, Y, Z \mid x y=y x, y z=z y, x z=z x(x \in X, y \in Y, z \in Z]$ and their graphs will be presented. The graphs obtained are related to the word length and the exponent sum. The exponent sum of $X, Y$ and $Z$ are one, two and $n(n \in \mathbf{N})$ respectively. Finally, the number of generator of the diagram group can be determined.
\end{abstract}

\section{INTRODUCTION}

Diagram group was introduced by Meakin and Sapir in 1993. Their student, Kilibarda obtained the first result about diagram group in his thesis [8], (see [9]). Further result about diagram group were discussed for example in Ahmad [1], Ahmad and Al-Odhari [2], Guba and Sapir [6, 7] and Farley [4].

Let $\mathcal{S}=[X \mid R]$ be a semigroup presentation. Here $X$ is an alphabet set while elements of $R$ are of the form $R_{\varepsilon}=R_{-\varepsilon}$ where $R_{ \pm \varepsilon}$ are positive word on $X$ are of the form $x_{1} x_{2} \ldots x_{n}\left(x_{i} \in X\right)$

Received 17 June 2006, revised 27 November 2006, accepted 3 November 2007. 

below:

We define an atomic picture $\mathbf{A}=\left(W_{1} R_{\varepsilon} W_{2}, W_{1} R_{-\varepsilon} W_{2}\right)$ over $S$ as an object

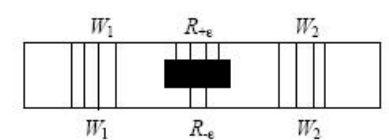

The initial and terminal of $\mathbf{A}$ is given by $\iota(\mathbf{A})=W_{1} R_{\varepsilon} W_{2}$ and $\tau(\mathbf{A})=$ $W_{1} R_{-\varepsilon} W_{2}$ respectively. Thus we can relate $\mathbf{A}$ as an edge

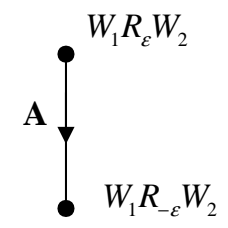

of a graph.

Suppose that $\mathbf{A}, \mathbf{B}$ are two atomic picture such that $\tau(\mathbf{A})=\iota(\mathbf{B})$. Then we my define the composition $\mathbf{A} \circ \mathbf{B}$ as a picture

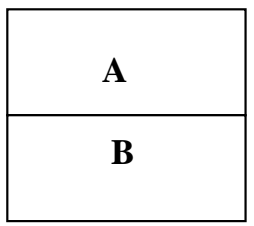

or as a graph can be written as

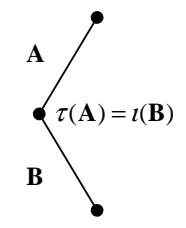

Collection of all possible composition of atomic pictures can be written as a connected graph such that the edges are atomic pictures and vertices are all possible positive words on $\mathbf{X}$. As a graph, we may obtain the fundamental group, denoted by $D(S)$.(Refer $[8])$. This group is called the diagram group. 
In this paper, we will discuss about the diagram groups of direct product of three free semigroups generated by $X, Y$, and $Z$. Thus the presentation given by

$$
\mathcal{S}=[X, Y, Z \mid x y=y x, y z=z y, x z=z x(x \in X, y \in Y, z \in Z]
$$

Wang [10] would like to know the properties of diagram groups for semidirect product in order to determine the finite set of generating pictures of the group. This is a continuation of our work in [5] where we consider the direct product of two free semigroup.

Let $W$ be positive word in $X \bigcup Y \cup Z$. The exponent sum of $X$ in $W, \sigma_{X} W$, is defined to be the number of element set $X$ appear in $W$. The exponent sum of $Y$ in $W, \sigma_{Y}(W)$ and the exponent sum of $Z$ in $W, \sigma_{Z}(W)$ are defined in similar manner. We will be proof

\section{Theorem 1:}

Let $\mathcal{S}=[X, Y, Z \mid x y=y x, y z=z y, x z=z x(x \in X, y \in Y, z \in Z]$ be a semigroup presentation and $W$ is positive word in set $X \bigcup Y \bigcup Z$. If $\sigma_{X} W=1, \sigma_{Y}(W)=1$ and $\sigma_{Z}(W)=n$, then diagram group $D(\mathcal{S})$ is isomorphic to $\mathbf{Z}^{n^{2}}$

\section{Theorem 2:}

Let $\mathcal{S}=[X, Y, Z \mid x y=y x, y z=z y, x z=z x(x \in X, y \in Y, z \in Z]$ be a semigroup presentation and $W$ is positive word in set $X \bigcup Y \bigcup Z$. If $\sigma_{X} W=1, \sigma_{Y}(W)=2$ and $\sigma_{Z}(W)=n$, then the graph of diagram group $D(\mathcal{S})$ contains

$$
\frac{n^{3}+6 n^{2}+11 n+6}{2}
$$

vertices and

$$
\frac{3 n^{3}+11 n^{2}+12 n+4}{2}
$$

edges. The diagram group $D(\mathcal{S})$ is isomorphic to $\mathbf{Z}^{k}$, where

$$
k=\frac{2 n^{3}+5 n^{2}+n}{2} .
$$

To proof Theorem 1 and Theorem 2, we can use the following lemma of Cohen [3].

Lemma 1: Let $\Gamma$ be connected graph with $n$ vertices. If $n$ is finite then $\pi_{1}(\Gamma)$ is isomorphic to $\mathbf{Z}^{k}, k=m-n+1$ 


\section{PROOF OF THEOREM 1}

Let $W=x y z_{1} z_{2} \ldots z_{n}, x \in X, y \in Y, z_{i} \in Z$. Then $\sigma_{X}(W)=1, \sigma_{Y}(W)=1$ and $\sigma_{Z}(W)=n(n \in \mathbf{N})$. Hence the graph $\Gamma_{\mathcal{S}}$ contains vertex $W$ is as follows

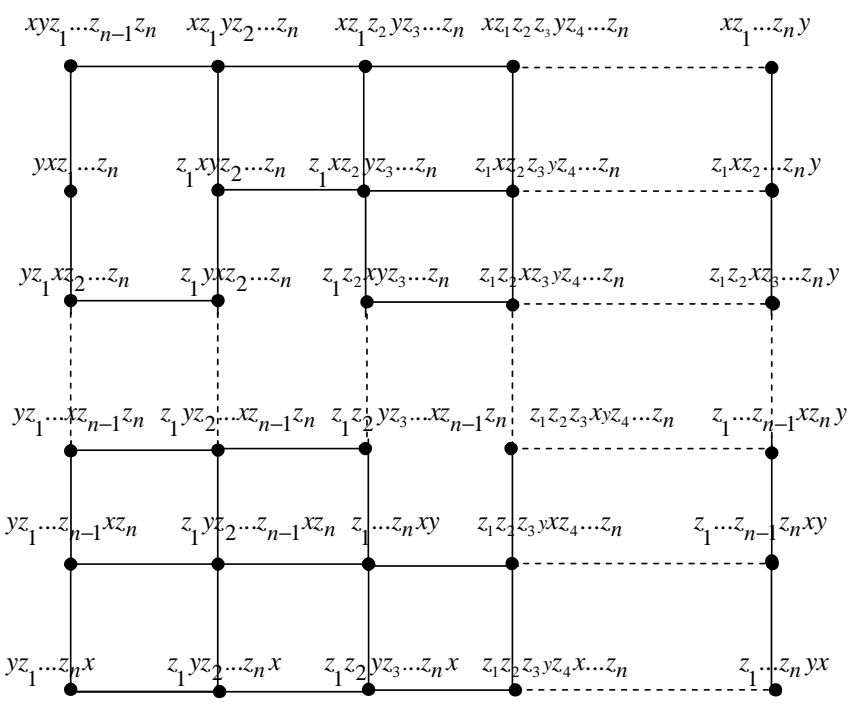

From the graph above,the number of vertices is $(n+2)(n+1)$ and the number of edges is $(n+1)(2 n+1)$. Note that for all vertices with labelled word $W$ such that $\sigma_{X}(W)=1, \sigma_{Y}(W)=1$ and $\sigma_{Z}(W)=n$ are connected. Using Lemma 1, we have $D(\mathcal{S})$ is isomorphic to $\mathbf{Z}^{n^{2}}$

\section{PROOF OF THEOREM 2.}

Let $W=x y_{1} y_{2} z_{1} z_{2} \ldots z_{n}, x \in X, y \in Y, z_{i} \in Z$ and hence $\sigma_{X}(W)=1$, $\sigma_{Y}(W)=2$ and $\sigma_{Z}(W)=n(n \in \mathbf{N})$.

Then we obtain the graph as follow 


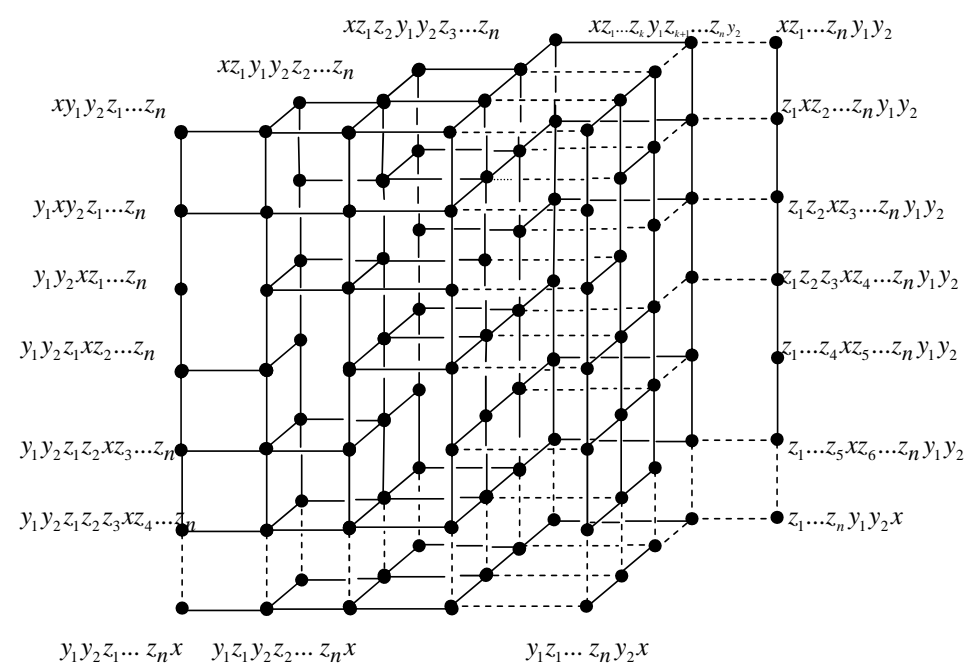

From the graph above, we have the number of vertices is

$$
(n+3)\left[3+\sum_{j=1}^{n-1}(j+2)\right]=\frac{n^{3}+6 n^{2}+11 n+6}{2}
$$

and the number of edges is

$$
\left.(n+2) \sum_{j=1}^{n-2}(3 j+5)+(2 n+8)\right]=\frac{3 n^{3}+11 n^{2}+12 n+4}{2}
$$

As in the proof of theorem 1 for all vertices with labelled $W$ such that $\sigma_{X}(W)=1$, $\sigma_{Y}(W)=2$ and $\sigma_{Z}(W)=n$, and are connected.

Using Lemma 1 , we have $D(\mathcal{S})$ is isomorphic to $\mathbf{Z}^{k}$, where

$$
k=\frac{2 n^{3}+5 n^{2}+n}{2} .
$$

Acknowledgement. This work was supported by Pemda Provinsi Riau under PHD program grant 


\section{REFERENCES}

1. A.G. Ahmad, Triviality Problems for Diagram Groups. Jour. of Inst. of Math. \& Comp.Sc. (Math. Ser.), Vol. 16(2), (2003),105-107.

2. A.G. Ahmad \& A.M. Al-Odhari, The Graph of Diagram Groups Constructud from Natural Numbers Semigroup with a Repeating Generators. Jour. of Inst. of Math. \& Comp. Sc. (Math. Ser.), Vol 17(1),(2004), 67-68.

3. D.E. Cohen, Combinatorial Group Theory a Topological Approach. LMS Students Text 14 Cambridge, (1989), cup

4. D.S. Farley, Finiteness and CAT(0) Properties of Diagram Groups, Topologi, 42, (2003), 1065-1082.

5. Sri Gemawati and A.G. Ahmad, Diagram Groups from Direct Product Two Free Semigroups of Length Two, (2005). Technical Report.

6. V. Guba and M. Sapir, Diagram Groups, Memoirs of the AMS 130, (1997), No.620.

7. V.Guba and M.Sapir, Rigidity Properties of Diagram Groups, Int. J. of Algebra and Compt, Vol.12(2), (2002), 9-17.

8. V. Kilibarda, On the Algebra of Semigroup Diagrams, PhD. Thesis, (1994), University of Nebraska-Lincoln.

9. V. Kilibarda, On the Algebra of Semigroup Diagrams. Int. J. of Algebra and Compt, Vol. 7(3), (1997), 313-338.

10. J. Wang, Finite derivation type for semi-direct products of monoid. Theoretical Computer Science, 191, (1998), 219-228.

Sri Gemawati : Department of Mathematics, Riau University, Pekanbaru, Indonesia.

E-mail: gemawati@yahoo.co.id

Abd. Ghafur Bin Ahmad: School of Mathematical Sciences, Faculty of Science and Technology, Universiti Kebangsaan Malaysia, Bangi 43600, Selangor D.E., Malaysia.

E-mail: ghafur@pkrisc.cc.ukm.my 